\title{
Mycobacterium ulcerans and Mycobacterium marinum: Pathogenesis, Diagnosis and Treatment
}

\author{
Loh Teng-Hern Tan ${ }^{1 *}$, Pendru Raghunath ${ }^{2}$, Long Chiau Ming ${ }^{3}$, Jodi Woan-Fei Law ${ }^{1 *}$ \\ ${ }^{1}$ Novel Bacteria and Drug Discovery (NBDD) Research Group, Microbiome and Bioresource Research Strength (MBRS), \\ Jeffrey Cheah School of Medicine and Health Sciences, Monash University Malaysia, 47500 Bandar Sunway, Selangor \\ Darul Ehsan, Malaysia. \\ ${ }^{2}$ Department of Microbiology, School of Medicine, Texila American University, Georgetown, Guyana. \\ ${ }^{3}$ PAPRSB Institute of Health Sciences, Universiti Brunei Darussalam, Jalan Tungku Link Gadong BE1410, Brunei \\ Darussalam.
}

\begin{abstract}
Skin and soft tissue infections are common presentations for non-tuberculous mycobacteria (NTM). The cutaneous infections caused by NTM may cause localized or diffuse lesions. M. ulcerans is one of the most identified pathogens that involves in the skin and soft tissue mycobacterial infections. Meanwhile, M. marinum, as an NTM has also become important emerging causal agents of cutaneous disease in various geographical regions. Although having common ancestry and highly similar in genetic makeup, M. ulcerans and M. marinum have differential impacts on the host innate immune system. In term pathogenesis, prolonged cell exposure to exotoxin mycolactone produced by M. ulcerans could lead to Buruli ulcer. Meanwhile, like most pathogenic mycobacteria, M. marinum evades the host immune responses by invading and replicating inside host cells and it is capable of modulating host immune responses. This article aims to provide a general overview and comparisons between the pathogenesis, diagnosis, prevention and therapeutic strategies for M. ulcerans and M. marinum.
\end{abstract}

Keywords: Mycobacterium ulcerans; Mycobacterium marinum; diagnosis; pathogenesis; treatment

*Correspondence: Loh Teng-Hern Tan, Jeffrey Cheah School of Medicine and Health Sciences, Monash University Malaysia, 47500, Bandar Sunway, Selangor Darul Ehsan, Malaysia; loh. teng.hern@monash.edu. Jodi Woan-Fei Law, Jeffrey Cheah

Received: $17^{\text {th }}$ August 2020 School of Medicine and Health Sciences, Monash University

Accepted: $18^{\text {th }}$ September 2020

Published Online: $30^{\text {th }}$ September 2020 Malaysia, 47500, Bandar Sunway, Selangor Darul Ehsan, Malaysia; jodi.law1@monash.edu.

Citation: Tan LTH, Raghunath P, Ming LC et al. Mycobacterium ulcerans and Mycobacterium marinum: Pathogenesis, diagnosis and treatment. Prog Microbes Mol Biol 2020; 3(1): a0000114. https://doi.org/10.36877/pmmb.a0000114

\section{INTRODUCTION}

Mycobacteria are a group of aerobic, acid-fast bacteria which are slender, non-flagellated and rod in shape. They possess waxy cell wall composed of mycolic acid, enabling them to be resistant to decolorization even with the use of acidified alcohol. The acid-fastness property of mycobacteria can be demonstrated by the ZiehlNeelsen stain $^{[1]}$. In general, the genus Mycobacterium is broadly categorized into three main groups which include the Mycobacterium tuberculosis complex (MTC), Mycobacterium leprae and non-tuberculous mycobacteria (NTM). Being the two major human pathogens, $M$. tuberculosis and M. leprae cause tuberculosis and leprosy, respectively.

Unlike the other two groups which are pathogenic to human, NTM, or atypical mycobacteria, encompasses a variety species which commonly inhabit the aquatic and terrestrial environments. More than 170 species of NTM has been discovered and the list keeps increasing. These mycobacteria form biofilm that contributes to their survival in diverse ecological niches ${ }^{[2]}$, including soil, water (such as household water) plants, animals and food products. Although NTM disease is not notifiable in most countries, the rise in the prevalence of NTM disease has become a growing health concern in the recent years. The reasons include the aging of the population, the increasing number of immunocompromised patients and the increased awareness of the disease. The NTM can cause pulmonary as well as extrapulmonary diseases (lymphadenitis, cutaneous disease, disseminated disease), that often inflicting immunocompromised individual and patients with pre-existing conditions. Generally, NTM infections are acquired from environmental exposures via inhalation (e.g. aerosol) or inoculation (e.g. trauma, plastic surgery, acupuncture) $[3,4]$.

Skin and soft tissue infection is one of the common 
presentations for NTM. Mycobacteria responsible for most skin disease include $M$. ulcerans, M. marinum, M. chelonae, M. fortuitum, M. avium-intracellulare, M. tuberculosis and M. leprae. The cutaneous diseases caused by mycobacteria usually manifest as nodules with characteristics of crusting, ulcers and hypo- and hyperpigmentation. Furthermore, cutaneous infections associated with these mycobacteria may cause localized or diffuse lesions. M. ulcerans is one of the most identified pathogens that involves in the skin and soft tissue mycobacterial infections. Meanwhile, $M$. marinum, has also become the emerging pathogens causing cutaneous diseases in people from various countries. Although having a common ancestry and they are highly similar in terms of genetic makeup, M. marinum and M. ulcerans exhibit differential impacts on the innate host immune system. The production of mycolactone plays a main role of M. ulcerans in the pathogenesis of Buruli ulcer disease. Meanwhile, M. marinum is similar to most pathogenic mycobacteria where the bacteria evade the host immune responses by invading and replicating inside host cells and are capable to modulate host immune responses (Figure 1).

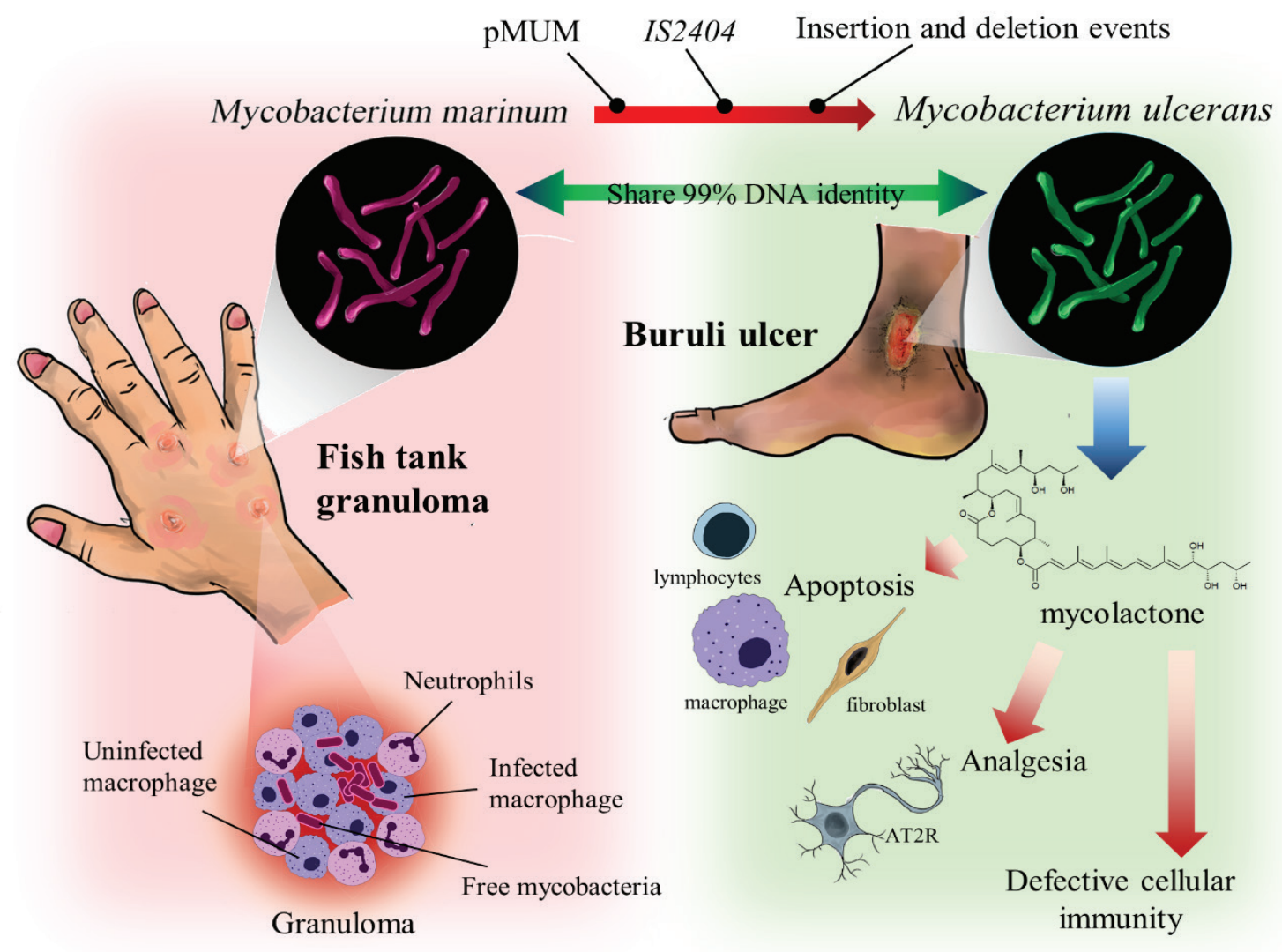

Figure 1. The differential clinical presentations and the pathogenesis between M. marinum and M. ulcerans despite they share high genomic similarity.

The aim of this review is to provide an overview on both $M$. ulcerans and M. marinum as the two major human pathogenic mycobacteria species commonly implicated in cutaneous diseases. Besides that, the pathogenesis and diagnosis of $M$. ulcerans and M. marinum infections are summarized and compared. The prevention and ideal strategies to control the diseases are also discussed in this review.

\section{MYCOBACTERIUM MARINUM VERSUS MYCOBACTERIUM ULCERANS}

Both M. marinum and M. ulcerans are known to be the two opportunistic mycobacterial pathogens, also named as the mycolactone-producing mycobacteria (MPM), that secrete plasmid-encoded mycolactone exotoxins ${ }^{[5]}$. Mycolactone is a cytotoxic polyketide metabolite produced by MPM, essential for bacterial virulence, to induce Buruli ulcer-like lesions characterized by extensive necrosis and void of inflammation in intradermal administered animal models ${ }^{[6]}$. The mycolactone molecule is known to cause apoptosis of mammalian cells, especially more toxic toward the anchorage-dependent cells leading to cytoskeletal rearrangements and detachment in in vitro experiments. Interestingly, studies also indicate that mycolactone alters the primary role of innate immunity, including immune cells trafficking and TLR-induced cytokine production.

M. marinum was first isolated from salt water dead fish by Aronson (1926) ${ }^{[7]}$ and was considered as an opportunistic human pathogen after its retrieval from granulomatous skin lesions from Swedish swimmers in the year of $1951^{[8]}$. M. marinum is categorized under the Runyon's Group I photochromogenic NTM that are commonly found in nonchlorinated fresh or salt water ${ }^{[9]}$. Being an opportunistic human pathogen, $M$. marinum causes zoonotic infection in individuals who had exposed through direct-contact with the bacterium from fishes, especially when handling the contaminated aquariums ${ }^{[10]}$. In general, M. marinum infections manifest as superficial skin infections that marked by granuloma and lymphangitis. Thus, the infection of $\mathrm{M}$. 
marinum is also termed as the 'fish tank granuloma' or 'aquarium granuloma'. Meanwhile, more severe infections that can spread in a sporotrichoid pattern $^{[11]}$ to deeper tissue inflicting tendinitis, arthritis and osteomyelitis may occur especially in immunocompromised host ${ }^{[12,13]}$.

M. ulcerans is the causative pathogen of a neglected tropical disease, Buruli disease, being one of the most common mycobacterial disease worldwide after tuberculosis and leprosy ${ }^{[14]}$. Buruli disease is a chronic, necrotizing skin disease with cutaneous tissue destruction and large ulcerations. The Buruli disease cases primarily occur in central Africa, and other regions, including Asia, South America, the western Pacific and Australasia ${ }^{[15]} . M$. ulcerans strains possess a large circular virulence plasmid named pMUM which contains 3 genes encoding polyketide synthases $(m l s A 1, m l s A 2$ and $m l s B)$ responsible for the synthesis of the lipid toxin mycolactone ${ }^{[16]}$. Interestingly, $M$. ulcerans is genetically closely related to the $M$. marinum, thereby they share $99 \%$ DNA identity in which M. ulcerans exhibit reduced genomes. The comparative whole genomic studies suggest that the emergence of $M$. ulcerans has recently evolved from a M. marinum progenitor via acquisition of the virulence plasmid pMUM and subsequent reductive evolution ${ }^{[16]}$. Acquisition of this plasmid has been considered to be the main contributor for Buruli ulcer in humans ${ }^{[17]}$. The reduced genome of $M$. ulcerans was subjected to substantial gene loss due to DNA deletion s, DNA rearrangements mediated by insertion of IS2404 and IS2606 elements for niche adaptation ${ }^{[18,19]}$.

Both M. ulcerans and M. marinum strains have optimum growth temperature around $32^{\circ} \mathrm{C}^{[20]}$, but they grow poorly at $37^{\circ} \mathrm{C}$ and above, thus reflecting the preference of both strains for the skin and their limited systemic dissemination ${ }^{[21]}$. Considering both pathogens belonging to the group of slow-growing mycobacteria, M. marinum has longer doubling time than $M$. ulcerans when grown in microbial culture medium ${ }^{[22]}$. In Australia, a mean incubation period of four and half months was identified for M. ulcerans infections ${ }^{[23]}$. Meanwhile, the incubation period of $M$. marinum was approximately $\sim 3$ weeks but can be prolonged up to 9 months prior to symptoms onset $^{[24]}$. Nevertheless, both $M$. marinum and M. ulcerans infections may resolve by host immune responses while long-term antibiotic therapy is required on an established infection.

\section{DIAGNOSIS OF M. ULCERANS AND M. MARINUM}

Rapid identification and differentiation of Mycobacterium species are crucial to determine the appropriate therapeutic regimens. However, a definitive diagnosis of cutaneous mycobacterial infections can be challenging to make in the clinical routine. There are several diagnostic methods available include histology, microbiological cultures and molecular detection. Histologically, M. marinum infections manifest non-specific acute or chronic inflammation as well as positive for tuberculous granulomas and abscesses. However, the detection of acid-fast rod-like bacteria is unusual. Meanwhile, M. ulcerans infections are associated with septal subcutaneous necrosis of adiposerich tissue and positive for acid-fast bacteria detection ${ }^{[25]}$.
The conventional microbiological detection of mycobacteria can be done in specific solid or liquid medium to assist in devising successful antibiotic regimens. The cultivation of NTM is greatly varied depending on the type of pathogen. Incubation of both solid and liquid media at both $30^{\circ} \mathrm{C}$ and $35^{\circ} \mathrm{C}$ are usually done to optimally recover the NTM. Different media compositions and conditions according based on specific NTM metabolic needs are also required for their isolation, hence the suspected mycobacterial species could be suggested by the clinicians based on source of exposure and the clinical symptoms to the microbiologists ${ }^{[25]}$.

Being one of the most commonly used molecular-based method, polymerase chain reaction (PCR) has been used for rapid detection of pathogens based on specific target DNA sequence ${ }^{[26-28]}$. Specific polymerase chain reaction (PCR) assays are available and have been established as the gold standard for the identification and discrimination of NTM that representing public health hazard ${ }^{[29]}$. PCR can be performed on different clinical samples, including swabs, punch biopsies and fine needle aspirates from nodules, plaques as well as edematous lesions that have not ulcerated yet. Several target genes or sequences are commonly used to differentiate Mycobacterium sp., including the $16 S r R N A$, internal transcribed spacer (ITS), 23S rRNA, hsp-65, recA and $r p o B$ genes. Among the different genes, PCR targeting the insertion element IS2404 has shown to be highly sensitive and specific for M. ulcerans detection as it is present in high copy numbers ( $>200$ copies) in the $M$. ulcerans genome ${ }^{[30]}$ Timothy. A highly specific real-time qPCR assay was demonstrated to confer enhanced sensitivity of 10 -folds higher than the IS2404 PCR and provide quantitative assessment of $M$. ulcerans dissemination in Buruli ulcer lesions $^{[31]}$. Meanwhile, molecular detection technique for M. marinum is limited due to its high homology with $M$. ulcerans. Typically, molecular identification of $M$. marinum was performed by analysing $16 \mathrm{~S}$ rRNA or other conserved gene related to a week-growth of photochromogenic colonies $^{[29]}$.

Although PCR has high sensitivity, sophisticated laboratory infrastructure and well-trained personnel are required to obtain reliable PCR assays with strict quality control ${ }^{[32]}$. Meanwhile, these criteria are not available in endemic communities. Recently, loop-mediated amplification (LAMP), an isothermal amplification technique, has been proposed as an efficient diagnostic tool for detection of M. ulcerans. Being a promising alternative to PCR, this technique offers readily readable results within a short turnaround time and without the need of a thermocycler, hence extending molecular diagnosis in fieldwork and at the point of care ${ }^{[33]}$.

\section{PATHOGENESIS OF M. ULCERANS AND $M$. MARINUM}

M. ulcerans causes an ulcerative skin infection, namely Buruli ulcer, which is a destructive infection of subcutaneous tissues that result in ulcerative lesions in skin, soft tissue and even the bone. M. ulcerans differs from many other mycobacteria in term of its implications for pathology and immune response in human. M. ulcerans is found to be distributed extracellularly around the coagulative necrosis regions which is different from other mycobacteria that are 
intracellular macrophage pathogens. This observation led to early proposal that $M$. ulcerans produces an exotoxin $^{[34]}$. The cytotoxic molecule 'mycolactone' was successfully isolated and purified from the acetone soluble fraction of lipid extracts of $M$. ulcerans in $1999^{[35]}$ Kathleen. Principally, the pathogenesis of $M$. ulcerans is mediated by the production of mycolactone which is uncommon among other bacterial exotoxins. Mycolactones consist of a group poorly immunogenic polyketide-derived macrolides that have strong cytotoxic effects against most of the immune cells and skin cells. There are different variants of mycolactone molecules have been identified ${ }^{[36,37]}$. The typical mycolactone $\mathrm{A} / \mathrm{B}$ occurs in Africa while mycolactone $\mathrm{C}$ is found in Australia. In in vitro, mycolactone $\mathrm{A} / \mathrm{B}$ is more toxic than type $C$, the clinical significance of these differences remains elusive.

The paramount role of mycolactone in the pathogenesis of M. ulcerans was first established via the administration of the purified mycolactone into the skin of experimental animals which resulted in cell death but devoid of acute inflammatory response ${ }^{[38]}$. The major role of mycolactone in Buruli ulcer pathogenesis was further fortified by the infection of laboratory animals with $M$. ulcerans mutants which lack of mycolactone production. In contrary to the extracellular infection induced by the wild-type M. ulcerans, an intracellular inflammatory infection identical to that of $M$. marinum $^{[39]}$ was resulted by the mycolactone-negative mutants ${ }^{[40,41]}$.

Mycolactone has three major adverse implications on the host in mediating the pathogenesis of M. ulcerans infection. The chief destructive outcome of mycolactone is its apoptosis and necrosis inducing effects on an array of cells, including the immune cells. Mve-Obiang et al. (2003) ${ }^{[42]}$ revealed the potent cytotoxic effect of mycolactone $\mathrm{A} / \mathrm{B}$, as low as $0.1 \mathrm{ng} / \mathrm{mL}$ was sufficient to induce cell death associated with apoptosis ${ }^{[38]}$ and necrosis ${ }^{[43]}$. Recently, mycolactone was demonstrated to induce Bim-dependent cell apoptosis via the $\mathrm{mTORC}_{2}$ Akt-FoxO axis $^{[44]}$. Secondly, a down-regulation of overall host immune defence due to the impairments on the production of tumor necrosis factor (TNF) and other secretory proteins via the blockade of Sec61 by mycolactone. Sec61 is a heterotrimeric complex responsible for the transport of all secretory and integral transmembrane proteins into the endoplasmic reticulum in eukaryotic cell. The blockade of Sec61 activity affects the production of interferon-gamma (IFN- $\gamma$ ) and IFN- $\gamma$ in activated lymphocytes as well as nitric oxide synthase production in macrophages ${ }^{[45]}$. Thus, an effective immune response is failed to be activated by the host to act upon the underlying mycobacterial infection. Thirdly, mycolactone also causes impairment of pain sensitivity by targeting the type 2 angiotensin II receptors (AT2R) to mediate its analgesic effect. The mycolactone was suggested to induce analgesia by direct cytotoxicity against sensory neurons and Schwann cells, hence resulting in nerve damage ${ }^{[46,47]}$.

As for M. marinum, this bacterium causes tuberculosislike infection in the ectotherms and induces caseating granulomas in zebrafish that are similar to those in humans ${ }^{[48]}$. M. marinum is an opportunistic intracellular pathogen that multiplies in non-acid phagosome of macrophages prior to phagolysosome fusion ${ }^{[49]}$. Within the cells, $M$. marinum acquires the ability to escape from the phagosome into the cytoplasm to actively stimulate actinpolymerization, resulting to direct spread into adjacent cells via actin-based motility. The translocation of $M$. marinum into the host cell cytosol depends on an intact Region-of-Difference-1-locus (RD1) which encodes a Type-VII secretion system (ESX-1) that plays a role in mycobacterial virulence ${ }^{[50,51]}$. Thus, this mechanism confers immune evasion for $M$. marinum by spreading from cell to cell, contributing to permanent infection. M. marinum was further found to employ the nonlytic spreading mechanism where the mycobacterium is ejected from the cell via the ejectosome, a F-actin based structure, enabling the transmission to naïve host macrophages ${ }^{[52]}$. Then, the macrophages migrate into deeper tissue, where they start to form the pathological granuloma-like aggregates after phagocytosis of $M$. marinum. Moreover, M. marinum was shown to harbour the ESX-5 system of mycobacteria that responsible for the production of various prolineproline-glutamic acid (PPE) and proline-glutamic acid (PE)-polymorphic GC-rich repetitive sequence (PGRS) proteins. These proteins were demonstrated to interact with host immune system and evade the innate immune response via antigenic variation ${ }^{[53]}$, thus contributing to persistent infection ${ }^{[54,55]}$. For instance, the expression of PPE38 protein on the cell wall of $M$. marinum was shown to involve in bacterial surface properties and proinflammatory effects on infected macrophages ${ }^{[56]}$.

In the view of granulomas as host-beneficial protective structures that has long been a tenet of medical and immunology textbooks, studies employing the zebrafish embryo model of $M$. marinum infection have challenged the idea and provided evidence that the granulomas may be harnessed by mycobacteria for their dissemination and proliferation $^{[57,58]}$. The study revealed that $M$. marinum employs the ESX-1-dependent early macrophage aggregate to promote spread and growth ${ }^{[57]}$. On top of that, the mature and established granulomas are found to be porous, where newly infecting mycobacteria can infiltrate and persist within $^{[59]}$.

\section{PREVENTION AND TREATMENT STRATEGIES}

An utmost priority to curb Buruli ulcer disease is to enhance our knowledge on the transmission pathway of M. ulcerans to human that could aid in the preventive measures focusing on early detection and administration of effective treatment. However, the greatest challenge in Buruli ulcer control is that the reservoir and transmission of M. ulcerans are unclear. Exposure to water sources near endemic villages has been shown to increase the risk for developing Buruli ulcer, but it is a challenge to reduce the exposure, particularly in children, to such sources in rural West Africa ${ }^{[60]}$. The development of an effective vaccine to confer protection has enormous significance in areas of high endemicity for Buruli ulcer. However, there is no effective vaccine specifically targeting M. ulcerans is available clinically. The Bacillus Calmette-Guérin (BCG) 
vaccine is the only licensed vaccine against mycobacterial infections approved clinically that used to prevent tuberculosis. Although the BCG is cross-protective against M. ulcerans, it has only been associated with delaying the onset of disease and short-lived protection in small trials. Collectively, outreach programs to educate communities in endemic areas to recognize early stage of Buruli ulcer is extremely crucial for prevention of severe forms of the disease.

Although there are no vaccine and effective protective strategies, antimicrobial therapy has showcased effective treatment of the disease and lowered the recurrence rate. Given that single-drug treatment led to relapse of mycobacterial disease due to the emergence of drugresistant mycobacterial strains, multi-drug treatment regimens have been employed for mycobacterial infections. It is a common phenomenon that drug-resistant mutants repopulate the lesions following monotherapy, especially in both tuberculosis ${ }^{[61]}$ and leprosy ${ }^{[62]}$. Therefore, a second companion drug should be combined with the highly active core antimicrobial agent to prevent treatment failure and relapse.

In 2004, WHO recommended a combination antibiotics alone for small early lesion or as an adjunct to surgical resection for large lesions ${ }^{[63]}$. A randomised controlled trial reported similar efficacy between the use of either rifampicin and streptomycin ( 8 weeks) or rifampicin and streptomycin (4 weeks) followed by rifampicin and clarithromycin (4 weeks) which resulted in high recovery rates of exceeding $90 \%$ for patients inflicted with early $\left(<6\right.$ months) and small lesion $(<10 \mathrm{~cm})^{[64]}$. Recently, a fully oral rifampicin and an extended release formulation of clarithromycin has shown comparable effectiveness for treatment of early and limited Buruli ulcer ${ }^{[65]}$.

According to Center of Disease Control and Prevention, the public water facilities such as swimming pools, spas and hot tub are advised to maintain adequate concentrations of free chlorine, ranging between 0.4 to $1 \mathrm{mg} /$ liter in swimming pool and 2 to $5 \mathrm{mg} /$ liter for spas and hot tub ${ }^{[6,67]}$. Frequent sanitation and disinfection, and removal of infected fishes are the main control strategies of $M$. marinum infection in fishes. The maintenance personnel for the aquarium should use waterproof gloves to prevent any potential upper limb skin lesions exposure to the pathogen during fish tankrelated activities. Proper training is also essential for high-risk populations, such as fishermen and marine-life handlers, to identify signs of $M$. marinum in fish or human in order to facilitate more prompt treatment ${ }^{[68]}$.

To date, there is no clinical trial available which could suggest optimal management of $M$. marinum infections. Furthermore, there is no standardized treatment for cutaneous infections due to $M$. marinum, the therapeutic choice is mainly based on the severity of the infection and the immunocompetency of the patient ${ }^{[9]}$. Principally, rapid recovery from $M$. marinum in man requires the proper treatment and prevent further progression to deeper tissues. Based on retrospective case studies, single agent antibiotic therapy was shown to successfully treat majority of the limited cutaneous $M$. marinum infections. These single antibiotic agents include minocycline, doxycycline, cotrimoxazole-trimethoprim and clarithromycin have demonstrated positive outcomes in the treatment of $M$. marinum infections ${ }^{[9,69]}$. Besides that, the combination use of 2 active agents including ethambutol, clarithromycin/ azithromycin or rifampicin for 3 to 4 months has been reported to be effective adjunct therapy together with surgical debridement for invasive $M$. marinum infections ${ }^{[70]}$. Other antimicrobials used for treatment of M. marinum infection include ciprofloxacin, moxifloxacin, isoniazid and protionamide ${ }^{[71]}$. Nevertheless, there were reported cases that yielded negative therapeutic outcomes ${ }^{[72,73]}$. Several reports also described the worsening of $M$. marinum infection in patients receiving anti-TNF- $\alpha$ therapy ${ }^{[74,75]}$. Thus, it should be recommended to halt the use of TNF- $\alpha$ inhibitor or other immunosuppressive therapy in $M$. marinum infected patients who are under the course of antibiotics.

Surgical debridement remains a controversial therapy option for M. marinum infection and it should be limited to cases that fulfil certain criteria, including cases that associated with poor prognosis involving deep lesions, persistent drainage of sinus and chronic pain ${ }^{[76]}$. There are also other therapeutic modalities such as local hyperthermic therapy, photodynamic therapy, electrodessication, cryotherapy and X-ray therapy have been recommended to treat $M$. marinum infection ${ }^{[9]}$. Bacteriophage therapy represents an interesting strategy to be developed for the management of $M$. marinum infection despite only phage therapy using mycobacteriophage D29 for treatment of Buruli ulcer is available at the moment ${ }^{[77]}$.

\section{CONCLUSION}

Research on both $M$. ulcerans and $M$. marinum is vital for much needed advancement in the prevention and management of Buruli ulcer and fish tank granuloma, which are both challenging diseases that have been largely neglected. A clearer view of the exact innate and adaptive immune mechanisms leading to protection from $M$. ulcerans and $M$. marinum infections will greatly propel the development of new strategies for effective vaccine design. Moreover, future research focusing on clinical applications and epidemiology is essential to advance our knowledge of mycobacterial pathogens that cause cutaneous infection and improve our capability to control and treat these infections with optimal medical interventions.

\section{Authors Contributions}

The literature review and manuscript writing were performed by LT-HT and JW-FL. PR and LC-M provided vital insight and performed proof-reading. The research project is conceptualized by LT-HT and JW-FL.

\section{Conflict of Interest}

The authors declare that there is no conflict of interest in this work. 


\section{Reference}

1. Sethuraman G, Dev T, and Ramesh V. Mycobacterial Skin Infections. Harper's Textbook of Pediatric Dermatology 2019; 485-502.

2. Esteban J and García-Coca M. Mycobacterium biofilms. Front Microbiol 2018; 8: 2651

3. Lyman MM, Grigg C, Kinsey CB, et al. Invasive nontuberculous mycobacterial infections among cardiothoracic surgical patients exposed to heater-cooler devices. Emerg Infect Dis 2017; 23(5): 796.

4. Buser GL, Laidler MR, Cassidy PM, et al. Outbreak of Nontuberculous Mycobacteria Joint Prosthesis Infections, Oregon, USA, 2010-2016. Emerg Infect Dis 2019; 25(5): 849.

5. Li W-T, Chang H-W, Pang VF, et al. Mycolactone-producing Mycobacterium marinum infection in captive Hong Kong warty newts and pathological evidence of impaired host immune function. Dis Aquat Organ 2017; 123(3): 239-249.

6. Gehringer M and Altmann K-H. The chemistry and biology of mycolactones. Beilstein J Org Chem 2017; 13(1): 1596-1660.

7. Aronson JD. Spontaneous tuberculosis in salt water fish. J Infect Dis 1926 ; $315-320$.

8. Nordén $\AA$ and Linell F. A new type of pathogenic mycobacterium. Nature 1951; 168(4280): 826-826.

9. Rallis E and Koumantaki-Mathioudaki E. Treatment of Mycobacterium marinum cutaneous infections. Expert Opin Pharmacother 2007; 8(17): 2965-2978.

10. Petrini B. Mycobacterium marinum: Ubiquitous agent of waterborne granulomatous skin infections. Eur J Clin Microbiol Infect Dis 2006; 25(10): 609-613.

11. Fleming P and Keystone JS. Mycobacterium marinum infection with sporotrichoid spread from fish tank exposure. Can Med Assoc J 2017; 189(2): E76-E76.

12. Holden IK, Kehrer M, Andersen AB, et al. Mycobacterium marinum infections in Denmark from 2004 to 2017: A retrospective study of incidence, patient characteristics, treatment regimens and outcome. Sci Rep 2018; 8(1): 1-7.

13. Sia TY, Taimur S, Blau DM, et al. Clinical and pathological evaluation of Mycobacterium marinum group skin infections associated with fish markets in New York City. Clin Infect Dis 2016; 62(5): 590-595.

14. Portaels F, Silva MT, and Meyers WM. Buruli ulcer. Clin Dermatol 2009; 27(3): 291-305.

15. Simpson H, Deribe K, Tabah EN, et al. Mapping the global distribution of Buruli ulcer: A systematic review with evidence consensus. Lancet Glob Heatlh 2019; 7(7): e912-e922.

16. Stinear TP, Hong H, Frigui W, et al. Common evolutionary origin for the unstable virulence plasmid pMUM found in geographically diverse strains of Mycobacterium ulcerans. J Bacteriol 2005; 187(5): 1668-1676.

17. Demangel C, Stinear TP, and Cole S. Buruli ulcer: Reductive evolution enhances pathogenicity of Mycobacterium ulcerans. Nat Rev Microbiol 2009; 7(1): 50-60.

18. Stinear TP, Seemann T, Pidot S, et al. Reductive evolution and niche adaptation inferred from the genome of Mycobacterium ulcerans, the causative agent of Buruli ulcer. Genome Res 2007; 17(2): 192-200.

19. Yip MJ, Porter JL, Fyfe JAM, et al. Evolution of Mycobacterium ulcerans and other mycolactone-producing mycobacteria from a common Mycobacterium marinum progenitor. J Bacteriol 2007; 189(5): 2021-2029.

20. Eddyani M and Portaels F. Survival of Mycobacterium ulcerans at 37 C. Clin Microbiol Infect 2007; 13(10): 1033-1035

21. Feldman WH, Karlson AG, and Herrick J. Mycobacterium ulcerans: Pathogenesis of infection in mice, including determinations of dermal temperatures. Am J Pathol 1957; 33(6): 1163.

22. Marsollier L, Sévérin T, Aubry J, et al. Aquatic snails, passive hosts of Mycobacterium ulcerans. Appl Environ Microbiol 2004; 70(10): 6296-6298.

23. Loftus MJ, Trubiano JA, Tay EL, et al. The incubation period of Buruli ulcer (Mycobacterium ulcerans infection) in Victoria, Australia - Remains similar despite changing geographic distribution of disease. PLoS Negl Trop Dis 2018; 12(3): e0006323.

24. Jernigan JA and Farr BM. Incubation period and sources of exposure for cutaneous Mycobacterium marinum infection: Case report and review of the literature. Clin Infect Dis 2000; 31(2): $439-443$

25. Kromer C, Fabri M, Schlapbach C, et al. Diagnosis of mycobacterial skin infections. J Dtsch Dermatol Ges 2019; 17(9): 889-893.

26. Cheah Y-K, Salleh NA, Lee L-H, et al. Comparison of PCR fingerprinting techniques for the discrimination of Salmonella enterica subsp. enterica serovar Weltevreden isolated from indigenous vegetables in Malaysia. World J Microb Biot 2008; 24(3): 327

27. Khoo C-H, Cheah Y-K, Lee L-H, et al. Virulotyping of Salmonella enterica subsp. enterica isolated from indigenous vegetables and poultry meat in Malaysia using multiplex-PCR. Antonie Van Leeuwenhoek 2009; 96(4): 441

28. Law JW-F, Ab Mutalib N-S, Chan K-G, et al. Rapid methods for the detection of foodborne bacterial pathogens: Principles, applications, advantages and limitations. Front Microbiol 2015; 5: 770 .
29. Chemlal K, Huys G, Fonteyne P-A, et al. Evaluation of PCR-restriction profile analysis and IS2404 restriction fragment length polymorphism and amplified fragment length polymorphism fingerprinting for identification and typing of Mycobacterium ulcerans and M. marinum. J Clin Microbiol 2001; 39(9): 3272-3278.

30. Stinear T, Ross BC, Davies JK, et al. Identification and characterization of IS2404 and IS2606: Two distinct repeated sequences for detection of Mycobacterium ulcerans by PCR. J Clin Microbiol 1999; 37(4): 1018-1023.

31. Rondini S, Mensah-Quainoo E, Troll H, et al. Development and application of real-time PCR assay for quantification of Mycobacterium ulcerans DNA. J Clin Microbiol 2003; 41(9): 4231-4237.

32. Eddyani M, Lavender C, de Rijk WB, et al. Multicenter external quality assessment program for PCR detection of Mycobacterium ulcerans in clinical and environmental specimens. PLoS One 2014; 9(2): e89407.

33. Frimpong M, Ahor HS, Wahed AAE, et al. Rapid detection of Mycobacterium ulcerans with isothermal recombinase polymerase amplification assay. PLoS Negl Trop Dis 2019; 13(2): e0007155.

34. Connor D and Lunn H. Buruli ulceration. A clinicopathologic study of 38 Ugandans with Mycobacterium ulcerans ulceration. Arch Pathol 1966; 81(3): 183-199

35. George KM, Chatterjee D, Gunawardana G, et al. Mycolactone: A polyketide toxin from Mycobacterium ulcerans required for virulence. Science 1999; 283(5403): 854-857.

36. Van Der Werf TS, Barogui YT, Converse PJ, et al. Pharmacologic management of Mycobacterium ulcerans infection. Expert Rev Clin Pharmacol 2020; 13(4): 391-401.

37. Kim H-J and Kishi Y. Total synthesis and stereochemistry of mycolactone F. J Am Chem Soc 2008; 130(6): 1842-1844.

38. George KM, Pascopella L, Welty DM, et al. A Mycobacterium ulcerans toxin, mycolactone, causes apoptosis in guinea pig ulcers and tissue culture cells. Infect Immun 2000; 68(2): 877-883.

39. Doig KD, Holt KE, Fyfe JA, et al. On the origin of Mycobacterium ulcerans, the causative agent of Buruli ulcer. BMC Genomics 2012; 13(1): 258 .

40. Coutanceau E, Marsollier L, Brosch R, et al. Modulation of the host immune response by a transient intracellular stage of Mycobacterium ulcerans: The contribution of endogenous mycolactone toxin. Cell Microbiol 2005; 7(8): 1187-1196.

41. Adusumilli S, Mve-Obiang A, Sparer T, et al. Mycobacterium ulcerans toxic macrolide, mycolactone modulates the host immune response and cellular location of M. ulcerans in vitro and in vivo. Cell Microbiol 2005; 7(9): 1295-1304.

42. Mve-Obiang A, Lee RE, Portaels F, et al. Heterogeneity of mycolactones produced by clinical isolates of Mycobacterium ulcerans: implications for virulence. Infect Immun 2003; 71(2): 774-783.

43. Hong $\mathrm{H}$, Demangel $\mathrm{C}$, Pidot SJ, et al. Mycolactones: Immunosuppressive and cytotoxic polyketides produced by aquatic mycobacteria. Nat Prod Rep 2008; 25(3): 447-454.

44. Bieri R, Scherr N, Ruf M-T, et al. The macrolide toxin mycolactone promotes Bim-dependent apoptosis in Buruli ulcer through inhibition of mTOR. ACS Chem Biol 2017; 12(5): 1297-1307.

45. Baron L, Paatero AO, Morel J-D, et al. Mycolactone subverts immunity by selectively blocking the Sec61 transloconImmunity lost in translocation. J Exp Med 2016; 213(13): 2885-2896.

46. Anand U, Sinisi M, Fox M, et al. Mycolactone-mediated neurite degeneration and functional effects in cultured human and rat DRG neurons: Mechanisms underlying hypoalgesia in Buruli ulcer. Mol Pain 2016; 12: 1744806916654144

47. En J, Kitamoto S, Kawashima A, et al. Mycolactone cytotoxicity in Schwann cells could explain nerve damage in Buruli ulcer. PLoS Negl Trop Dis 2017; 11(8): e0005834.

48. Tobin DM and Ramakrishnan L. Comparative pathogenesis of Mycobacterium marinum and Mycobacterium tuberculosis. Cell Microbiol 2008; 10(5): 1027-1039.

49. Barker LP, George KM, Falkow S, et al. Differential trafficking of live and dead Mycobacterium marinum organisms in macrophages. Infect Immun 1997; 65(4): 1497-1504.

50. Houben D, Demangel C, van Ingen J, et al. ESX-1-mediated translocation to the cytosol controls virulence of mycobacteria. Cell Microbiol 2012; 14(8): 1287-1298.

51. Smith J, Manoranjan J, Pan M, et al. Evidence for pore formation in host cell membranes by ESX-1-secreted ESAT-6 and its role in Mycobacterium marinum escape from the vacuole. Infect Immun 2008; 76(12): 5478-5487.

52. Gerstenmaier L, Pilla R, Herrmann L, et al. The autophagic machinery ensures nonlytic transmission of mycobacteria. Proc Natl Acad Sci U S A 2015; 112(7): E687-E692.

53. Dong D, Wang D, Li M, et al. PPE38 modulates the innate immune response and is required for Mycobacterium marinum virulence. Infect Immun 2012; 80(1): 43-54.

54. Chan K, Knaak T, Satkamp L, et al. Complex pattern of Mycobacterium marinum gene expression during long-term granulomatous infection. Proc Natl Acad Sci 2002; 99(6): 3920-3925.

55. Hodgkinson JW, Ge J-Q, Grayfer L, et al. Analysis of the immune response in infections of the goldfish (Carassius auratus L.) with Mycobacterium marinum. Dev Comp Immunol 2012; 38(3): 456-465.

56. Weerdenburg EM, Abdallah AM, Mitra S, et al. ESX-5-deficient Mycobacterium marinum is hypervirulent in adult zebrafish. Cell Microbiol 2012; 14(5): 728-739.

57. Volkman HE, Clay H, Beery D, et al. Tuberculous granuloma formation is enhanced by a mycobacterium virulence determinant. PLoS Biol 2004; 2(11): e367.

58. Swaim LE, Connolly LE, Volkman HE, et al. Mycobacterium 
marinum infection of adult zebrafish causes caseating granulomatous tuberculosis and is moderated by adaptive immunity. Infect Immun 2006; 74(11): 6108-6117.

59. Cosma CL, Humbert O, and Ramakrishnan L. Superinfecting mycobacteria home to established tuberculous granulomas. Nat Immunol 2004; 5(8): 828-835.

60. Wansbrough-Jones MH and Phillips RO. 63 - Buruli Ulcer, in Hunter's Tropical Medicine and Emerging Infectious Diseases. 10th ed. E.T. Ryan, et al., Editors. 2020, Elservier: London. p. 565-568.

61. Caminero JA, Scardigli A, van der Werf T, et al. Treatment of drugsusceptible and drug-resistant tuberculosis. Tuberculosis (ERS Monograph). Sheffield, European Respiratory Society 2018: 152-178.

62. Scollard DM, Adams L, Gillis T, et al. The continuing challenges of leprosy. Clin Microbiol Rev 2006; 19(2): 338-381.

63. Organization WH and Initiative GBU. Provisional guidance on the role of specific antibiotics in the management of Mycobacterium ulcerans disease (Buruli ulcer). 2004, World Health Organization.

64. Nienhuis WA, Stienstra Y, Thompson WA et al Antimicrobial treatment for early, limited Mycobacterium ulcerans infection: randomised controlled trial. Lancet 2010; 375(9715): 664-672.

65. Phillips RO, Robert J, Abass KM, et al. Rifampicin and clarithromycin (extended release) versus rifampicin and streptomycin for limited Buruli ulcer lesions: A randomised, open-label, non-inferiority phase 3 trial. Lancet 2020; 395(10232): 1259-1267.

66. Division CfDCEHS. Swimming pools: Safety and disease control through proper design and operation. 1976: The Center.

67. Control CfD and Prevention. Suggested health and safety guidelines for public spa and hot tubs. Washington, DC: DHHS 1985; 15.

68. Bonamonte D, Filoni A, Vestita M, et al. Mycobacterium marinum Skin Infection, in Mycobacterial Skin Infections. D. Bonamonte an G. Angelini, Editors. 2017, Springer International Publishing: Cham. p. $325-358$

69. Aubry A, Chosidow O, Caumes E, et al. Sixty-three cases of Mycobacterium marinum infection: Clinical features, treatment, and antibiotic susceptibility of causative isolates. Arch Intern Med 2002; 162(15): 1746-1752.

70. Griffith DE, Aksamit T, Brown-Elliott BA, et al. An official ATS/IDSA statement: Diagnosis, treatment, and prevention of nontuberculous mycobacterial diseases. Am J Respir Crit Care Med 2007; 175(4): $367-$ 416.

71. Assiri A, Euvrard S, and Kanitakis J. Cutaneous Mycobacterium Marinum infection (fish tank granuloma) in a renal transplant recipient: Case report and literature review. Cureus 2019; 11(10): e6013-e6013.

72. Janik JP, Bang RH, and Palmer CH. Successful treatment of Mycobacterium marinum infection with minocycline after complication of disease by delayed diagnosis and systemic steroids. J Drugs Dermatol 2005; 4(5): 621-624.

73. Ljungberg B, Christensson B, and Grubb R. Failure of doxycycline treatment in aquarium-associated Mycobacterium marinum infections. Scand J Infect Dis 1987; 19(5): 539-543.

74. Chopra N, Kirschenbaum AE, and Widman D. Mycobacterium marinum tenosynovitis in a patient on etanercept therapy for rheumatoid arthritis JCR: Journal of Clinical Rheumatology 2002; 8(5): 265-268.

75. Harth M, Ralph E, and Faraawi R. Septic arthritis due to Mycobacterium marinum. J Rheumatol 1994; 21(5): 957.

76. Aubry A, Mougari F, Reibel F, et al. Mycobacterium marinum; p. 735 752 , in Tuberculosis and Nontuberculous Mycobacterial Infections. 7th ed. John Wiley \& Sons; 2017.

77. Trigo G, Martins TG, Fraga AG, et al. Phage therapy is effective against infection by Mycobacterium ulcerans in a murine footpad model. PLoS Negl Trop Dis 2013; 7(4): e2183. 